\title{
Noble Metal-Free Hierarchical ZrY Zeolite Efficient for Hydrogenation of Biomass-Derived Levulinic Acid
}

\author{
Di Hu, Hong Xu, Zuotong Wu, Man Zhang, Zhiyue Zhao, Yuchen Wang and Kai Yan*
}

Guangdong Provincial Key Laboratory of Environmental Pollution Control and Remediation Technology, School of Environmental Science and Engineering, Sun Yat-sen University, Guangzhou, China

\section{OPEN ACCESS}

Edited by:

Svetlana Ivanova,

University of Seville, Spain

Reviewed by: Jose Luis Santos Muñoz,

KU Leuven, Belgium Marcela Martínez Tejada, University of Seville, Spain

*Correspondence: Kai Yan

yank9@mail.sysu.edu.cn

Specialty section:

This article was submitted to Green and Sustainable Chemistry, a section of the journal Frontiers in Chemistry

Received: 15 June 2021 Accepted: 26 August 2021 Published: 12 October 2021

Citation: Hu D, Xu H, Wu Z, Zhang M, Zhao Z, Wang Y and Yan K (2021) Noble MetalFree Hierarchical ZrY Zeolite Efficient for Hydrogenation of Biomass-Derived

Levulinic Acid.

Front. Chem. 9:725175.

doi: 10.3389/fchem.2021.725175
Developing a low-cost and robust catalyst for efficient transformation of biomass-derived platform chemicals plays a crucial role in the synthesis of future transportation fuels. Herein, a post-synthetic strategy was employed to develop a noble metal-free and robust ZrY zeolite catalyst, which is efficient for the hydrogenation of biomass-derived levulinic acid (LA) into biofuel $\gamma$-valerolactone (GVL), whereas over 95\% yield of GVL was achieved in $10 \mathrm{~h}$ at $220^{\circ} \mathrm{C}$. The effects of acidic properties from ZrY catalysts and various reaction parameters on the catalytic performance were then discussed in detail. Subsequently, different characterization tools were used to compare the difference and relationship of structure activity between the fresh and spent ZrY catalysts. It was found that acidity and the metal-support interaction were important for the direct synthesis of GVL. This work provides a guideline to design a noble metal-free catalyst for high-value utilization of biomass-derived sources.

Keywords: noble metal-free, ZrY, hydrogenation, levulinic acid, gamma-valerolactone

\section{INTRODUCTION}

With the rapid consumption of fossil resources and the raising concern on the environmental issues, the development of efficient technologies for the conversion of renewable biomass into platform molecular and valuable chemicals has gained much attention around the world (Kunkes et al., 2008; Alonso et al., 2013; Wang et al., 2014). So far, various value-added chemicals (e.g., 5hydroxymethylfurfural, lactic acid, levulinic acid (LA), $\gamma$-valerolactone (GVL), and 2,5dimethylfuran) have been produced from naive biomass (Liu and Zhang, 2015; Xue et al., 2018). Among these valuable chemicals, GVL has been considered as a versatile platform chemical that can be used as a fuel additive, a solvent for biomass processing, and a precursor for the production of alkanes and valuable chemicals (Feng et al., 2019; Li et al., 2019; Winoto et al., 2019; Yu et al., 2019).

Over the last few decades, several methodologies for the production of GVL from various resources have been developed and reported (Moustani et al., 2018; Wang et al., 2018; Winoto et al., 2019). Among the various strategies, the heterogeneous hydrogenation of LA and its esters appeared more promising using various metal catalysts, such as noble metal (Pd (Yan et al., 2017a), Ru (Luo et al., 2014; Kuwahara et al., 2017), Au (Zhu et al., 2016), Pt (Luo et al., 2016), and Re (Yu et al., 2019)) and non-noble metal (Ni (Singh et al., 2018) and $\mathrm{Cu}$ (Hengne and Rode, 2012) ) catalysts. The noble metal catalysts seem to be the more active and selective candidates for the production of GVL from LA hydrogenation, due to their high intrinsic abilities in the activation of the $\mathrm{C}=\mathrm{O}$ bond (Luo et al., 2014; Kuwahara et al., 2017; Yan et al., 2017b). However, the high cost and complex synthesis production limit their practical application. Besides, the catalytic hydrogenation procedure often 
associates with the use of high $\mathrm{H}_{2}$ pressure, noble metals, and unsatisfied catalyst stability, restricting the actual utilizations. Developing noble metal-free candidates with high performance in the selective hydrogenation of LA is highly desired. Recently, Singh et al. (Singh et al., 2018) synthesized Ni/NiO catalyst for the catalytic hydrogenation of LA, and it was found that the acidity property plays an important role in the conversion of LA and ML to GVL with $94 \%$ yield. Zhang et al. (Zhang et al., 2018) reported that the geometric and electronic effects between $\mathrm{Cu}$ and $\mathrm{Ag}$ were responsible for the good activities of $\mathrm{CuAg} / \mathrm{Al}_{2} \mathrm{O}_{3}$ catalyst in the synthesis of GVL with approximately $100 \%$ yield. Recently, we also found the metal effect (Yi et al., 2020; Xu et al., 2021) and solvent effect (Xu et al., 2019) in tuning the hydrogenation of LA.

Although these abovementioned advanced works have been reported, few works have concentrated on the direct use of noble metal-free oxides for the hydrogenation of LA. On the other hand, the catalytic transfer hydrogenation of LA using Zr-based catalysts such as $\mathrm{ZrO}_{2}$ has been reported to effectively produce GVL, whereas $\mathrm{ZrO}_{2}$ was proposed as the active center ( $\mathrm{Li}$ et al., 2017). The strong metal-support interaction between $\mathrm{Ru}$ and nanotetragonal $\mathrm{ZrO}_{2}$ (Cao et al., 2017) made great contribution. The high porosity and acidity of the as-prepared catalyst facilitate the LA conversion to GVL. Zeolites were widely used in catalysis both as a catalyst and support due to the tunability of the structure and acidity. These findings inspired us to utilize the acidic property of $\mathrm{ZrO}_{2}$ and the strong metal-support interaction to overcome the considerable technical barrier.

Herein, we report the efficient hydrogenation of LA to GVL using the hierarchical noble metal-free $\mathrm{ZrY}$ catalysts $\left(\mathrm{ZrO}_{2}\right.$ supported on $\mathrm{NaY}$ ), whereas the $\mathrm{ZrY}$ catalyst was prepared using the post-synthetic method. Combination analysis tools were used to study the physical properties of these fabricated catalysts and understand the relationship between structure and activities. These fabricated catalysts were screened in the selective hydrogenation of LA, and various reaction parameters on the activities were investigated. Besides, catalyst stability was evaluated, and the proper reaction mechanism was proposed. In particular, the noble metal-free ZrY catalysts developed in this work are facile and alternative for the high-value upgrading of various biomass-derived chemicals.

\section{MATERIALS AND METHODS}

\section{Catalyst Preparation}

$\mathrm{NaY}$ with an Si/Al ratio of 5.4 was purchased from Nankai Catalyst Plant. $\mathrm{NaY}_{\text {meso }}$ was prepared by treating $\mathrm{NaY}$ which contains uniform micropores. Typically, $3.5 \mathrm{~g}$ of $\mathrm{NaY}$ and ethylenediamine-tetraacetic acid aqueous solution $(50 \mathrm{ml}$, $0.035,0.07$, and $0.105 \mathrm{~mol} \mathrm{~L}^{-1}$ ) were added to a flask. The mixture was refluxed and stirred at $373 \mathrm{~K}$ for $6 \mathrm{~h}$. Then, the solid product was filtered and dried for subsequent $\mathrm{NaOH}$ treatment. For the alkali treatment process, $1.7 \mathrm{~g}$ of solid powder was added to the $\mathrm{NaOH}$ aqueous solution $(50 \mathrm{ml}$, $0.4 \mathrm{~mol} \cdot \mathrm{L}^{-1}$ ) at $338 \mathrm{~K}$ for $0.5 \mathrm{~h}$. The formation of $\mathrm{NaY}_{\text {meso }}$ was then filtered, dried, and calcined in air at $823 \mathrm{~K}$ for $6 \mathrm{~h}$, and denoted as $\mathrm{Y}_{0.5}, \mathrm{Y}_{1}$, and $\mathrm{Y}_{1.5}$. In order to functionalize the

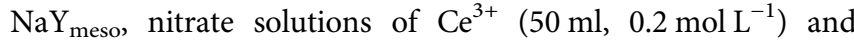
zirconium acetate $\left(50 \mathrm{ml}, 0.1,0.2\right.$, and $\left.0.3 \mathrm{~mol} \mathrm{~L}^{-1}\right)$ were, respectively, used to exchange $1.0 \mathrm{~g}$ of $\mathrm{NaY}_{\text {meso }}$ at $353 \mathrm{~K}$ for $12 \mathrm{~h}$. The filtered products were dried and calcined in air at $823 \mathrm{~K}$ for $6 \mathrm{~h}$, and then the powder denoted as $\mathrm{Ce}_{1} \mathrm{Y}_{1}$ and $\mathrm{Zr}_{\mathrm{x}} \mathrm{Y}_{\mathrm{y}}$ were obtained ( $\mathrm{x}$ and $\mathrm{y}$ was equal to $0.5,1$, and 1.5). Also, the synthesis procedures of hierarchical HZSM- 5 were referred to the previous literatures (Wang et al., 2017), and $\mathrm{Zr}_{1}$ HZSM-5 was produced from zirconium acetate $\left(50 \mathrm{ml}, 0.2 \mathrm{~mol} \mathrm{~L}^{-1}\right)$ treatment and calcination. $\mathrm{ZrY}$ was directly synthesized from $\mathrm{NaY}$ exchanging with zirconium acetate and calcination step. $\mathrm{ZrO}_{2}$ was obtained directly from the calcination of zirconium acetate at $823 \mathrm{~K}$ for $6 \mathrm{~h}$. All the reagents were bought from Macklin and used without any pretreatment. The used $\mathrm{Zr}_{1} \mathrm{Y}_{1}$ catalyst was recollected after five consecutive runs.

\section{Catalytic Tests}

The catalytic reaction was performed in an electrically heated high-pressure autoclave with a magnetic stirring system at $600 \mathrm{rpm}$, during $10 \mathrm{~h}$ at $220^{\circ} \mathrm{C}$ and $3 \mathrm{MPa} \mathrm{H}_{2}$, using $10 \mathrm{ml}$ of 1,4-dioxane, $4 \mathrm{mmol}$ of LA, and $100 \mathrm{mg}$ catalyst, and then the gas was released carefully after being cooled to room temperature. The reaction mixture was centrifuged for separation of slurry and supernatant. The slurry was washed several times with water and ethanol for a subsequent cyclic test and further analyzation; the supernatant was filtered through a $0.22-\mu \mathrm{m}$ syringe filter for analyzing by gas chromatography (Techcomp GC7900) equipped with a capillary column (TM-FFAP) and an FID detector.

The concentration of LA and GVL was calculated by standard calibration curves. The conversion of LA and selectivity of GVL were determined as follows:

LA Conversion $(\%)=1-\left(\frac{\text { Remaining amount of LA }}{\text { Initial amount of LA }}\right) \times 100 \%$,

GVL Selectivity $(\%)=\left(\frac{\text { Amount of GVL }}{\text { Sum amount of products }}\right) \times 100 \%$

\section{Catalyst Characterization}

X-ray diffraction (XRD) of all samples for the crystal phase analysis was recorded on a Rigaku Ultima IV diffractometer. Scanning electron microscope (SEM) images were obtained with a HitachiE-3500 to elucidate the morphologies of the samples. Transmission electron microscopy (TEM) images were obtained with an accelerating voltage of $300 \mathrm{kV}$ (TEM, TF20). Mapping was employed to explore the existence and the distribution of elements in the sample. Temperature-programmed desorption (TPD) experiments were performed using an AutoChem II 2920 V5.02 chemical adsorption instrument with a thermal conductivity detector (TCD). X-ray photoelectron spectra (XPS) were performed on an Escalab 250 X-ray photoelectron spectrometer (Thermo Fisher Scientific, USA). The thermal behavior of fresh and used catalysts were tested by TGA5500 from room temperature to $800^{\circ} \mathrm{C}$ under air atmosphere, with a 

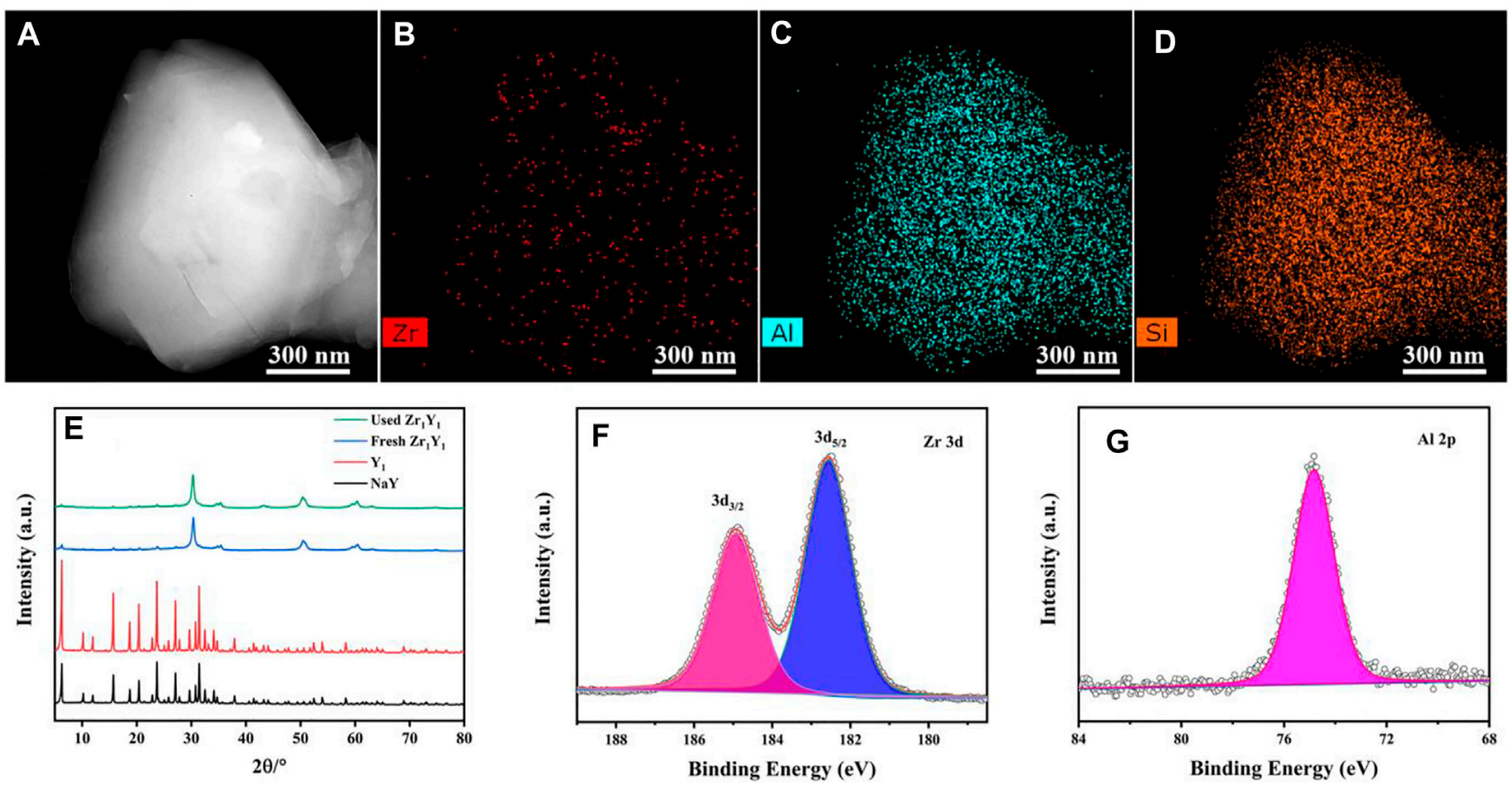

FIGURE 1 | TEM images of $Z r_{1} Y_{1}$ catalyst (A), corresponding EDS mapping patterns (B-D), XRD patterns of different samples (E), XPS spectra of the $Z r_{1} Y_{1}$ catalyst (F), $\mathrm{Zr} 3 \mathrm{~d}$, and (G) Al 2p.

heating rate of $10^{\circ} \mathrm{C} / \mathrm{min}$. Nitrogen sorption isotherms of samples were performed at $-196^{\circ} \mathrm{C}$ on Quantachrome instrument under nitrogen atmosphere.

\section{RESULTS AND DISCUSSION}

\section{Characterization Results of the Hierarchical ZrY Catalysts}

The characterization results for the TEM image and elemental distribution patterns of $\mathrm{Zr}, \mathrm{Al}$, and $\mathrm{Si}$ in the $\mathrm{Zr}_{1} \mathrm{Y}_{1}$ catalyst are presented in Figures 1A-D. The mapping results demonstrated the homogeneous dispersion of $\mathrm{Zr}$ (Figure 1B), $\mathrm{Al}$, and $\mathrm{Si}$ uniformly throughout the particles, indicating that $\mathrm{ZrO}_{2}$ was homogeneously immobilized onto the surface of the $\mathrm{NaY}_{\text {meso }}$ support with no severe aggregation. The effect of various parameters on the synthesis process (dealumination and $\mathrm{Zr}$ incorporation) on the $\mathrm{NaY}$ zeolite crystal structure was investigated by $\mathrm{XRD}$, as shown in Supplementary Figure S1 and Figure 1E. The treated $\mathrm{NaY}$ zeolite exhibited its inherent crystal structure, and the incorporation of $\mathrm{Zr}$ atoms into $\mathrm{NaY}_{\text {meso }}$ did not affect its crystal structure but displayed the similar XRD pattern as the parent $\mathrm{NaY}$. Importantly, there is apparently appearance of the typical tetragonal $\mathrm{ZrO}_{2}$ (JCPDS: 50-1,089) peaks at $30.3^{\circ}, 50.4^{\circ}$, and $60.2^{\circ}$ (Huang et al., 2018), suggesting the successful mobilization of $\mathrm{Zr}$ atoms onto the framework. In addition, there is no other type of diffraction peak for the $\mathrm{Zr}_{\mathrm{x}} \mathrm{Y}_{\mathrm{y}}$ catalyst, indicating the existence of $\mathrm{NaY}$ and $\mathrm{ZrO}_{2}$ for all samples. And there is a dilute effect in the adjusting of the $\mathrm{NaY}$ and $\mathrm{Zr}$ salt dosage. For the $\mathrm{Zr}_{1} \mathrm{Y}_{1.5}$ catalyst, the crystallinity was partially damaged, and the interaction between support and $\mathrm{ZrO}_{2}$ species was lower than that in the $\mathrm{Zr}_{1} \mathrm{Y}_{1}$ sample. Also, the fresh and used $\mathrm{Zr}_{1} \mathrm{Y}_{1}$ catalysts exhibited the same XRD patterns, indicating the strong interaction between $\mathrm{NaY}$ support and loaded $\mathrm{ZrO}_{2}$ species. Supplementary Figure S3 illustrated the XPS survey image, and Figures 1F,G displayed the XPS spectra of $\mathrm{Zr} 3 \mathrm{~d}$ and $\mathrm{Al} 2 \mathrm{p}$ in the $\mathrm{Zr}_{1} \mathrm{Y}_{1}$ catalysts. Regarding the $\mathrm{Zr} 3 \mathrm{~d}$ spectra, the $\mathrm{Zr}_{1} \mathrm{Y}_{1}$ catalyst exhibited two distinct peaks at 182.5 and $184.9 \mathrm{eV}$ corresponding

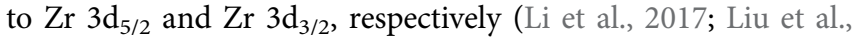
2017). These further confirmed the formation of the combining $\mathrm{ZrO}_{2}$ with stable zeolites in a single catalytic system.

The porosity information was analyzed by BET as shown in Supplementary Figure S4 and Supplementary Table S1. The dealuminized $\mathrm{Y}_{1}$ zeolite catalyst displays a typical type I adsorption isotherm superficial; from the Supplementary Figure S4 inset picture, a hysteresis loop can be seen, and an average diameter of $1.54 \mathrm{~nm}$ was obtained (Crisci et al., 2010), which is higher than that of $\mathrm{NaY}$, indicating the dealuminization effect. During the dealuminization process, the main structure was maintained, and the newly formed vacancy could act as the doping sites for the following step. After loading $\mathrm{Zr}$ onto the support, a type IV isotherm with $\mathrm{H} 4$ type hysteresis loops was recognized (Ahn et al., 2018), the specific surface area is $192 \mathrm{~m}^{2} / \mathrm{g}$, and the average pore size is $2.31 \mathrm{~nm}$, which shows clearly that the mesoporous structure existed. And this will facilitate the transportation of LA and GVL during the reaction process.

Ammonia temperature programmed desorption was further employed to investigate the acidity of $\mathrm{NaY}$ support, the asprepared catalyst, and used catalyst (Supplementary Figure S5) (Delidovich et al., 2014; Körner et al., 2018). The amount 
TABLE 1 | Acidity properties of $\mathrm{NaY}, \mathrm{Zr}_{1} \mathrm{Y}_{1}$, and used $Z r_{1} \mathrm{Y}_{1}$ catalysts.

\begin{tabular}{|c|c|c|c|c|c|c|}
\hline \multirow[t]{2}{*}{ Catalysts } & \multicolumn{2}{|c|}{ Weak acidity } & \multicolumn{2}{|c|}{ Moderate acidity } & \multicolumn{2}{|c|}{ Strong acidity } \\
\hline & $\mathbf{T}_{\mathrm{d}}\left({ }^{\circ} \mathbf{C}\right)$ & Amount (mmol/g) & $\mathbf{T}_{\mathrm{d}}\left({ }^{\circ} \mathrm{C}\right)$ & Amount (mmol/g) & $\mathbf{T}_{\mathrm{d}}\left({ }^{\circ} \mathrm{C}\right)$ & Amount $(\mathrm{mmol} / \mathrm{g})$ \\
\hline $\mathrm{NaY}$ & 153 & 2.59 & - & - & - & - \\
\hline$Z r_{1} Y_{1}$ & 123 & 0.68 & 276 & 0.68 & 593 & 0.33 \\
\hline Used $Z r_{1} Y_{1}$ & 100 & 0.16 & 337 & 0.92 & 547 & 0.06 \\
\hline
\end{tabular}

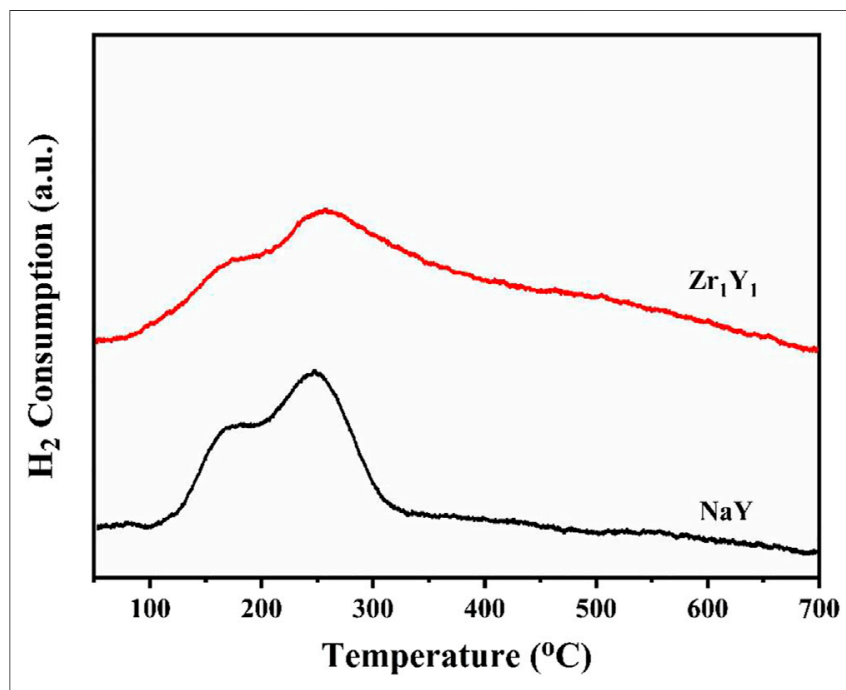

FIGURE 2 | $\mathrm{H}_{2}$-TPD profiles of the $\mathrm{NaY}$ and $\mathrm{Zr}_{1} \mathrm{Y}_{1}$ catalysts.

of different acidic sites was quantified and listed in Table 1. It can be found that $\mathrm{NaY}$ kept a desorption peak at around $153^{\circ} \mathrm{C}$, indicating the existence of weak acid sites, the acid amount was $2.59 \mathrm{mmol} / \mathrm{g}$, and there is no other type of acid sites detected. The desorption peak at around 276 and $593^{\circ} \mathrm{C}$ for the $\mathrm{Zr}_{1} \mathrm{Y}_{1}$ samples represented moderate and strong acid sites. Also, the used $\mathrm{Zr}_{1} \mathrm{Y}_{1}$ showed dominate acid sites are moderate and strong acid sites, with just $0.16 \mathrm{mmol} / \mathrm{g}$ weak acid sites. These results confirmed that the parent $\mathrm{NaY}$ only held weak acid sites. As our previous work reported (Xu et al., 2019; Yi et al., 2020), the acidity property plays an important role in the conversion of LA, and this work further confirmed that the moderate and strong acid sites can promote the hydrogenation of LA to GVL, whereas the weak acid sites have a weak ability to adsorb $\mathrm{H}_{2}$ and LA (Yan et al., 2015).

Figure 2 shows the $\mathrm{H}_{2}$ temperature programmed desorption $\left(\mathrm{H}_{2}-\mathrm{TPD}\right)$ profiles of the pristine and modified catalysts. For $\mathrm{Zr}_{1} \mathrm{Y}_{1}$ catalyst, the $\mathrm{H}_{2}$-TPD profile displays two $\mathrm{H}_{2}$ desorption peaks centered at 174 and $257^{\circ} \mathrm{C}$, respectively, in the range of $50-700^{\circ} \mathrm{C}$. The high-temperature peak was attributed to the $\mathrm{H}_{2}$ adsorbed in the subsurface and the spillover. The $\mathrm{Zr}_{1} \mathrm{Y}_{1}$ catalyst exhibits twice the $\mathrm{H}_{2}$ uptake ability than that of the pristine $\mathrm{NaY}$, which is 167 and $80.8 \mu \mathrm{mol} \mathrm{g}_{\mathrm{cat}}{ }^{-1}$, indicating that the $\mathrm{Zr}_{1} \mathrm{Y}_{1}$ is more efficient for the adsorption and dissociation sites of $\mathrm{H}_{2}$, which is further verified by the catalytic hydrogenation performance of the catalysts. The as-prepared $\mathrm{Zr}_{1} \mathrm{Y}_{1}$ catalyst displayed more easily accessible $\mathrm{ZrO}_{2}$ acidic sites and the

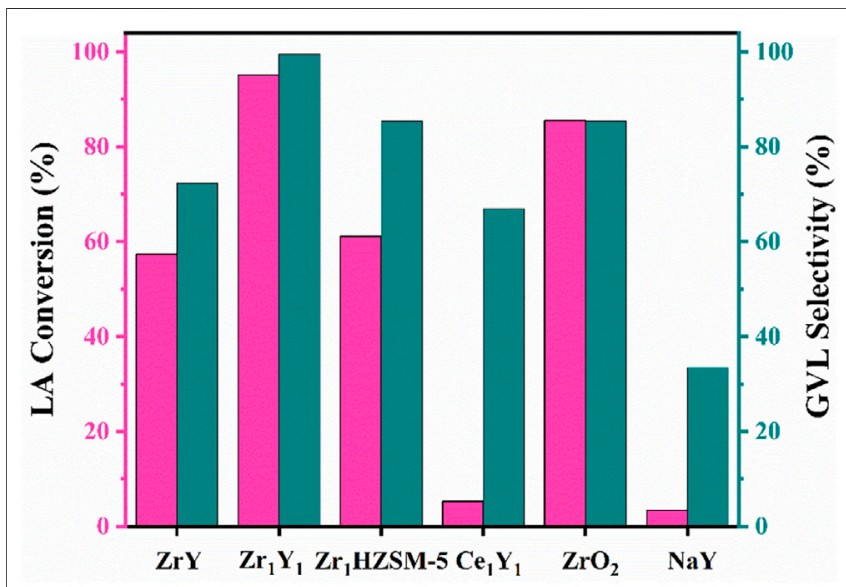

FIGURE 3 | Catalytic performance of LA hydrogenation with various catalysts.

higher affinity with the $\mathrm{H}_{2}$ resources, which is beneficial for the selective hydrogenation of LA in a bench reactor.

\section{Catalytic Hydrogenation of LA}

The as-prepared catalysts were emulated for the selective hydrogenation of LA under the same conditions. As revealed in Figure 3, $\mathrm{Zr}_{1} \mathrm{Y}_{1}$ catalyst exhibited the highest conversion of $95 \%$ and GVL selectivity of $99 \%$ among the presented catalyst. The hierarchical structure and suitable acidity offered outstanding transportation properties and active centers for the reaction. For example, it showed a negligible activity for the $\mathrm{NaY}$ support (less than 5\%), due to the lack of active sites. The sole usage of $\mathrm{ZrO}_{2}$ catalyst was also evaluated, and the hydrogenation reaction was quite active and can achieve $85 \%$; however, the activity in the second run decreased severely, which was probably owing to the strong adsorption ability in leading to the carbon deposit formation. When the acidic $\mathrm{ZrO}_{2}$ was directly loaded onto the microporous structure of $\mathrm{Y}$ zeolite by the exchanging process, $57 \%$ conversion of LA and $72 \%$ selectivity to GVL were obtained. Due to the lack of the modification step, a weak interaction between $\mathrm{ZrO}_{2}$ and $\mathrm{NaY}$ was formed. In contrast, the two different metals supported on hierarchical $\mathrm{Y}$ zeolite catalysts $\mathrm{Zr}_{1} \mathrm{Y}_{1}$ and $\mathrm{Ce}_{1} \mathrm{Y}_{1}$ displayed a thorough difference in the performance, and the $\mathrm{CeO}_{2}$ was unable to catalyze the conversion of LA. Also, another type of hierarchical zeolite HZSM-5 was chosen as a support for the synthesis of $\mathrm{Zr}_{1} \mathrm{HZSM}-5$ catalyst; apparently, the mesopore-contained 


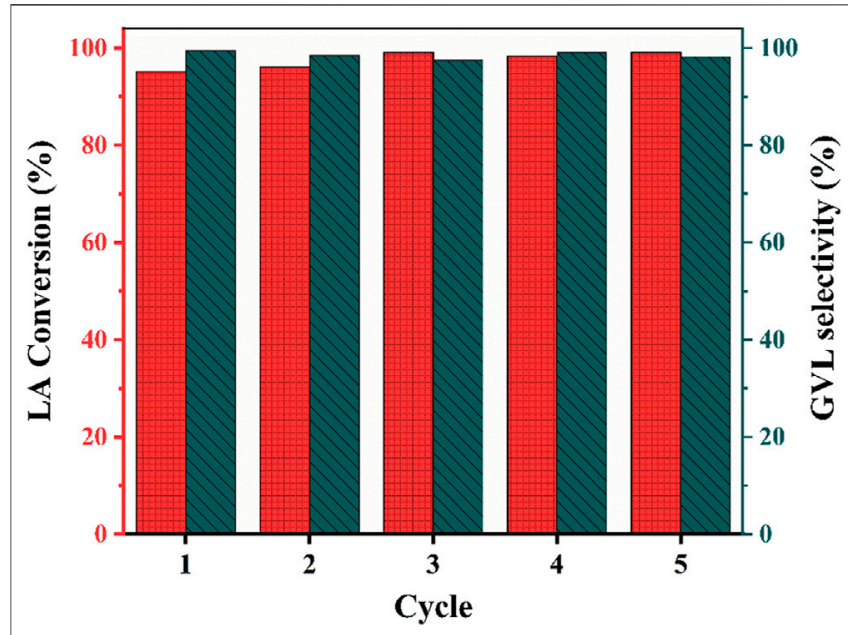

FIGURE 4 | Cyclic stability test of the $\mathrm{Zr}_{1} \mathrm{Y}_{1}$ catalyst.

$\mathrm{Zr}_{1}$ HZSM-5 catalyst could facilitate the hydrogenation reaction rate. These facts suggested that both the acidity of catalyst and the mesoporous structure can increase the catalytic activity in the direct hydrogenation of LA. Also, the strong interaction between metal and support is crucial for this process.

To optimize the catalytic performance of the modified $\mathrm{Zr}_{\mathrm{x}} \mathrm{Y}_{\mathrm{y}}$ catalyst in the selective hydrogenation of LA, the dealuminization rate and loading amount of $\mathrm{ZrO}_{2}$ varied, and their influence on the hydrogenation of LA is shown in Supplementary Figures S7,S8. When the dealuminization rate of $\mathrm{NaY}$ was changed from 50 to $150 \%$, the yield of GVL displayed a volcano-type behavior with a highest yield of GVL at $100 \%$, which is 73,95 , and $89 \%$, respectively. This can be attributed to the fact that the dealuminization rate of $\mathrm{NaY}$ matrix need to be controlled in a proper range. An enhancement in the dealuminization rate beyond $100 \%$ could lead to the breakdown of the NaY structure; this can be proved by the decrease in the intensity of $\mathrm{Zr}_{1} \mathrm{Y}_{1.5}$ in Supplementary Figure S1c. In another case, the optimum loading of $\mathrm{ZrO}_{2}$ on the hierarchical $\mathrm{NaY}_{\text {meso }}$ was found to be $\mathrm{Zr}_{1} \mathrm{Y}_{1}$. When the $\mathrm{ZrO}_{2}$ loading amount was lower than $100 \%$, the GVL yield decreased to $72 \%$. While the amount of $\mathrm{ZrO}_{2}$ was higher than $100 \%$, the GVL yield was $88 \%$, which also indicated the lack of active sites and pore blockage. Hence, an appropriate amount of the modification agent and $\mathrm{Zr}$ salt were used for the $\mathrm{Zr}_{1} \mathrm{Y}_{1}$ catalyst, and the changes in the physicochemical offer great support for the LA hydrogenation process.

Finally, the recyclability experiments were tested to evaluate the catalytic performance under the same reaction conditions. The cyclic results for the hydrogenation of LA to GVL using $\mathrm{Zr}_{1} \mathrm{Y}_{1}$ catalyst are shown in Figure 4, demonstrating that the LA hydrogenation and selectivity of GVL remained steadily even after five cycles. There is no decrease in all the runs, while it has $\sim 4.9 \%$ coke deposition formed as revealed in Supplementary Figure S6 (Zhao et al., 2018). For the zeolite type of catalyst, the coke deposition can be removed by the calcination step (Yi et al., 2020), and the $\mathrm{Zr}_{1} \mathrm{Y}_{1}$ catalyst was directly used after calcination at $550^{\circ} \mathrm{C}$ without any reducing treatment. Figure $\mathbf{1 E}$ displayed the XRD patterns of fresh and used $\mathrm{Zr}_{1} \mathrm{Y}_{1}$ catalysts, there are no obvious changes, indicating there is no severe $\mathrm{ZrO}_{2}$ species leaching process. In general, the $\mathrm{Zr}_{1} \mathrm{Y}_{1}$ catalyst was proven to be stable. Thus, the specific rate of reaction is about $4 \mathrm{mmol}_{\mathrm{GVL}} \mathrm{g}_{\text {cat }}{ }^{-1} \mathrm{~h}^{-1}$ in the consecutive runs. This was due to the $\mathrm{ZrO}_{2}$ uniform dispersed on the treated $\mathrm{Y}$ zeolite, effectively preventing agglomeration and enhancing the moderate and strong acidity of the weak acidity and micropore structure $\mathrm{Y}$ zeolite. And, with the reuse of the $\mathrm{Zr}_{1} \mathrm{Y}_{1}$ catalyst, the considerable amount of strong acidity was converted to moderate acidity due to the agglomeration process on the surface. In addition, the $\mathrm{Zr}_{1} \mathrm{Y}_{1}$ catalyst was directly separated from solutions with filtering and dried in the oven, and then it can be reused without any calcination, making it attractive for practical usage.

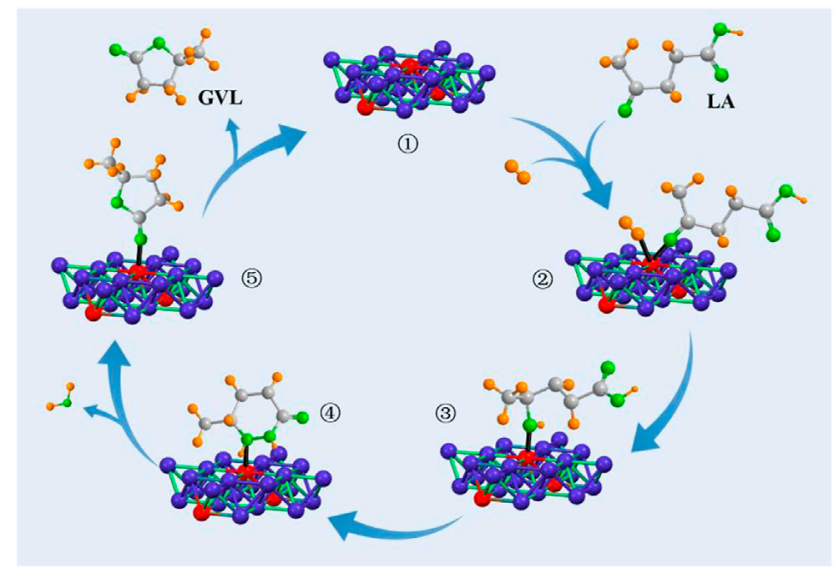

SCHEME 1 | Proposed mechanism for the direct hydrogenation of $L A$ to $G V L$ over the $Z r_{1} Y_{1}$ catalyst. 
Based on the catalytic performance, a plausible Meerwein-Ponndorf-Verley (MPV) reduction mechanism for the hydrogenation of LA to GVL was proposed through a catalytic cycle involving a six-member ring transition state. As shown in Scheme 1, molecular $\mathrm{H}_{2}$ and the ketone carbonyl group of LA were adsorbed to the $\mathrm{ZrO}_{2}$ sites. Then, 4-hydroxyvaleric acid was formed to generate state 2. Between intermediates 2 and 3, the two hydroxyl groups were linked together to create a six-membered ringlike structure, and ether was formed with the acidity catalyze, and 1 mole $\mathrm{H}_{2} \mathrm{O}$ was discharged during the reaction. After that, GVL and the bond between the $\mathrm{O}-\mathrm{O}$ linker were released. Each step in the whole process was believed to be reversible, and the reaction was driven by the thermodynamic properties of the intermediates (Wang et al., 2018). The newly formed 4-hydroxypentanoic was not stable and readily converted to target product GVL.

\section{CONCLUSIONS}

In summary, we have successfully developed noble metal-free $\mathrm{Zr}_{1} \mathrm{Y}_{1}$ catalysts by a post-synthetic strategy, which is efficient for the hydrogenation of LA into biofuel GVL. As a result, the conversion of LA and selectivity of GVL reached 95 and 99\%, respectively, under the reaction conditions of $220^{\circ} \mathrm{C}$ and $3 \mathrm{MPa} \mathrm{H}_{2}$ pressure in 1, 4dioxane solvent. The performance was comparable with previously reported candidates. Besides, it was found that acidic properties and the metal-support interaction play important roles in the synthesis of GVL. Moreover, these as-prepared $\mathrm{ZrY}$ catalysts maintained stable performance even over five consecutive runs, indicating the promising applications for the selective upgrading of other biomassderived chemicals.

\section{REFERENCES}

Ahn, S., Nauert, S. L., Buru, C. T., RimoldiChoi, M. H., Choi, H., Schweitzer, N. M., et al. (2018). Pushing the Limits on Metal-Organic Frameworks as a Catalyst Support: NU-1000 Supported Tungsten Catalysts for O-Xylene Isomerization and Disproportionation. J. Am. Chem. Soc. 140, 8535-8543. doi:10.1021/ jacs.8b04059

Alonso, D. M., Wettstein, S. G., and Dumesic, J. A. (2013). Gamma-valerolactone, a Sustainable Platform Molecule Derived from Lignocellulosic Biomass. Green. Chem. 15, 584-595. doi:10.1039/c3gc37065h

Cao, W., Luo, W., Ge, H., Su, Y., Wang, A., and Zhang, T. (2017). UiO-66 Derived Ru/ZrO2@C as a Highly Stable Catalyst for Hydrogenation of Levulinic Acid to $\gamma$-valerolactone. Green. Chem. 19, 2201-2211. doi:10.1039/c7gc00512a

Crisci, A. J., Tucker, M. H., Dumesic, J. A., and Scott, S. L. (2010). Bifunctional Solid Catalysts for the Selective Conversion of Fructose to 5-hydroxymethylfurfural. Top. Catal. 53, 1185-1192. doi:10.1007/s11244-010-9560-2

Delidovich, I., Leonhard, K., and Palkovits, R. (2014). Cellulose and Hemicellulose Valorisation: an Integrated challenge of Catalysis and Reaction Engineering. Energy Environ. Sci. 7, 2803-2830. doi:10.1039/c4ee01067a

Feng, H.-J., Li, X.-C., Qian, H., Zhang, Y.-F., Zhang, D.-H., Zhao, D., et al. (2019). Efficient and Sustainable Hydrogenation of Levulinic-Acid to Gamma-

\section{DATA AVAILABILITY STATEMENT}

The original contributions presented in the study are included in the article/Supplementary Material; further inquiries can be directed to the corresponding author.

\section{AUTHOR CONTRIBUTIONS}

Conceptualization, YK; writing-original draft preparation, $\mathrm{DH}$ and $\mathrm{ZW}$; data collection, $\mathrm{HX}, \mathrm{ZW}$, and ZZ; funding acquisition, $\mathrm{YK}, \mathrm{DH}$, MZ, and YW; writing-review and editing, YK. All authors have read and agreed to the published version of the manuscript.

\section{FUNDING}

This work was funded by the National Key R\&D Program of China (grant number 2018YFD0800700), National Ten Thousand Talent Plan, the National Natural Science Foundation of China (grant numbers 22078374 and 21776324), Key-Area Research and Development Program of Guangdong Province (grant number 2019B110209003), Guangdong Basic and Applied Basic Research Foundation (grant numbers 2019B1515120058 and 2020A1515011149), the Fundamental Research Funds for the Central Universities (grant number 19lgzd25), China Postdoctoral Science Foundation (grant number 2018M643310), and Hundred Talent Plan (grant number 201602) from Sun Yat-sen University.

\section{SUPPLEMENTARY MATERIAL}

The Supplementary Material for this article can be found online at: https://www.frontiersin.org/articles/10.3389/fchem.2021.725175/ full\#supplementary-material

Valerolactone in Aqueous Solution over Acid-Resistant CePO4/Co2P Catalysts. Green. Chem. 21, 1743-1756. doi:10.1039/c9gc00482c

Hengne, A. M., and Rode, C. V. (2012). Cu-ZrO2 Nanocomposite Catalyst for Selective Hydrogenation of Levulinic Acid and its Ester to $\gamma$-valerolactone. Green. Chem. 14, 1064-1072. doi:10.1039/c2gc16558a

Huang, Y.-B., Yang, T., Luo, Y.-J., Liu, A.-F., Zhou, Y.-H., Pan, H., et al. (2018). Simple and Efficient Conversion of Cellulose to $\gamma$-valerolactone through an Integrated Alcoholysis/transfer Hydrogenation System Using $\mathrm{Ru}$ and Aluminium Sulfate Catalysts. Catal. Sci. Technol. 8, 6252-6262. doi:10.1039/ c8cy01971a

Körner, P., Jung, D., and Kruse, A. (2018). The Effect of Different Brønsted Acids on the Hydrothermal Conversion of Fructose to HMF. Green. Chem. 20, 2231-2241. doi:10.1039/c8gc00435h

Kunkes, E. L., Simonetti, D. A., West, R. M., Serrano-Ruiz, J. C., Gartner, C. A., and Dumesic, J. A. (2008). Catalytic Conversion of Biomass to Monofunctional Hydrocarbons and Targeted Liquid-Fuel Classes. Science 322, 417-421. doi:10.1126/science.1159210

Kuwahara, Y., Kango, H., and Yamashita, H. (2017). Catalytic Transfer Hydrogenation of Biomass-Derived Levulinic Acid and its Esters to $\gamma$-Valerolactone over Sulfonic Acid-Functionalized UiO-66. ACS Sustain. Chem. Eng. 5, 1141-1152. doi:10.1021/acssuschemeng.6b02464

Li, S., Wang, Y., Yang, Y., Chen, B., Tai, J., Liu, H., et al. (2019). Conversion of Levulinic Acid to $\gamma$-valerolactone over Ultra-thin TiO2 Nanosheets Decorated 
with Ultrasmall $\mathrm{Ru}$ Nanoparticle Catalysts under Mild Conditions. Green. Chem. 21, 770-774. doi:10.1039/c8gc03529f

Li, W., Li, Y., Fan, G., Yang, L., and Li, F. (2017). Role of Surface Cooperative Effect in Copper Catalysts toward Highly Selective Synthesis of Valeric Biofuels. ACS Sustain. Chem. Eng. 5, 2282-2291. doi:10.1021/acssuschemeng.6b02618

Liu, B., and Zhang, Z. (2015). Catalytic Conversion of Biomass into Chemicals and Fuels over Magnetic Catalysts. ACS Catal. 6, 326-338. doi:10.1021/ acscatal.5b02094

Liu, S., Fan, G., Yang, L., and Li, F. (2017). Highly Efficient Transformation of $\gamma$-valerolactone to Valerate Esters over Structure-Controlled Copper/zirconia Catalysts Prepared via a Reduction-Oxidation Route. Appl. Catal. A: Gen. 543, 180-188. doi:10.1016/j.apcata.2017.06.032

Luo, W., Bruijnincx, P. C. A., and Weckhuysen, B. M. (2014). Selective, One-Pot Catalytic Conversion of Levulinic Acid to Pentanoic Acid over Ru/H-ZSM5. J. Catal. 320, 33-41. doi:10.1016/j.jcat.2014.09.014

Luo, Y., Yi, J., Tong, D., and Hu, C. (2016). Production of $\gamma$-valerolactone via Selective Catalytic Conversion of Hemicellulose in Pubescens without Addition of External Hydrogen. Green. Chem. 18, 848-857. doi:10.1039/c5gc01775k

Moustani, C., Anagnostopoulou, E., Krommyda, K., Panopoulou, C., Koukoulakis, K. G., Bakeas, E. B., et al. (2018). Novel Aqueous-phase Hydrogenation Reaction of the Key Biorefinery Platform Chemical Levulinic Acid into $\gamma$-valerolactone Employing Highly Active, Selective and Stable WaterSoluble Ruthenium Catalysts Modified with Nitrogen-Containing Ligands. Appl. Catal. B: Environ. 238, 82-92. doi:10.1016/j.apcatb.2018.07.009

Singh, H., Iyengar, N., Yadav, R., Rai, A., and Sinha, A. K. (2018). Facile Conversion of Levulinic Acid to $\gamma$-valerolactone Using a High Surface Area Magnetically Separable Ni/NiO Catalyst. Sustain. Energ. Fuels 2, 1699-1706. doi:10.1039/ c8se00274f

Wang, H., Chen, C., Zhang, H., Wang, G., and Zhao, H. (2018). An Efficient and Reusable Bimetallic Ni3Fe NPs@C Catalyst for Selective Hydrogenation of Biomass-Derived Levulinic Acid to $\gamma$-valerolactone. Chin. J. Catal. 39, 1599-1607. doi:10.1016/S1872-2067(18)63105-5

Wang, T., Nolte, M. W., and Shanks, B. H. (2014). Catalytic Dehydration of C6carbohydrates for the Production of Hydroxymethylfurfural (HMF) as a Versatile Platform Chemical. Green. Chem. 16, 548-572. doi:10.1039/ c3gc41365a

Wang, Y., Ma, J., Ren, F., Du, J., and Li, R. (2017). Hierarchical Architectures of ZSM-5 Nanocrystalline Aggregates with Particular Catalysis for Lager Molecule Reaction. Microporous Mesoporous Mater. 240, 22-30. doi:10.1016/ j.micromeso.2016.10.051

Winoto, H. P., Fikri, Z. A., Ha, J.-M., Park, Y.-K., Lee, H., Suh, D. J., et al. (2019). Heteropolyacid Supported on Zr-Beta Zeolite as an Active Catalyst for One-Pot Transformation of Furfural to $\gamma$-valerolactone. Appl. Catal. B: Environ. 241, 588-597. doi:10.1016/j.apcatb.2018.09.031

Xu, H., Hu, D., Yi, Z., Wu, Z., Zhang, M., and Yan, K. (2019). Solvent Tuning the Selective Hydrogenation of Levulinic Acid into Biofuels over Ni-Metal Organic Framework-Derived Catalyst. ACS Appl. Energ. Mater. 2, 6979-6983. doi:10.1021/acsaem.9b01439

Xu, H., Hu, D., Zhang, M., Wang, Y., Zhao, Z., Jiang, Z., et al. (2021). Bimetallic $\mathrm{NiCu}$ alloy Catalysts for Hydrogenation of Levulinic Acid. ACS Appl. Nano Mater. 4, 3989-3997. doi:10.1021/acsanm.1c00339
Xue, Z., Liu, Q., Wang, J., and Mu, T. (2018). Valorization of Levulinic Acid over Non-noble Metal Catalysts: Challenges and Opportunities. Green. Chem. 20, 4391-4408. doi:10.1039/c8gc02001a

Yan, K., Lafleur, T., Wu, X., Chai, J., Wu, G., and Xie, X. (2015). Cascade Upgrading of $\gamma$-valerolactone to Biofuels. Chem. Commun. 51, 6984-6987. doi:10.1039/ c5 5 c01463h

Yan, K., Liu, Y., Lu, Y., Chai, J., and Sun, L. (2017a). Catalytic Application of Layered Double Hydroxide-Derived Catalysts for the Conversion of BiomassDerived Molecules. Catal. Sci. Technol. 7, 1622-1645. doi:10.1039/ C7CY00274B

Yan, L., Yao, Q., and Fu, Y. (2017b). Conversion of Levulinic Acid and Alkyl Levulinates into Biofuels and High-Value Chemicals. Green. Chem. 19, 5527-5547. doi:10.1039/c7gc02503c

Yi, Z., Hu, D., Xu, H., Wu, Z., Zhang, M., and Yan, K. (2020). Metal Regulating the Highly Selective Synthesis of Gamma-Valerolactone and Valeric Biofuels from Biomass-Derived Levulinic Acid. Fuel 259, 116208. doi:10.1016/ j.fuel.2019.116208

Yu, Z., Lu, X., Liu, C., Han, Y., and Ji, N. (2019). Synthesis of $\gamma$-valerolactone from Different Biomass-Derived Feedstocks: Recent Advances on Reaction Mechanisms and Catalytic Systems. Renew. Sustain. Energ. Rev. 112, 140-157. doi:10.1016/j.rser.2019.05.039

Zhang, L., Mao, J., Li, S., Yin, J., Sun, X., Guo, X., et al. (2018). Hydrogenation of Levulinic Acid into Gamma-Valerolactone over In Situ Reduced CuAg Bimetallic Catalyst: Strategy and Mechanism of Preventing $\mathrm{Cu}$ Leaching. Appl. Catal. B: Environ. 232, 1-10. doi:10.1016/j.apcatb.2018.03.033

Zhao, P., Ye, L., Sun, Z., Lo, B. T. W., Woodcock, H., Huang, C., et al. (2018). Entrapped Single Tungstate Site in Zeolite for Cooperative Catalysis of Olefin Metathesis with Brønsted Acid Site. J. Am. Chem. Soc. 140, 6661-6667. doi:10.1021/jacs.8b03012

Zhu, S., Xue, Y., Guo, J., Cen, Y., Wang, J., and Fan, W. (2016). Integrated Conversion of Hemicellulose and Furfural into $\gamma$-Valerolactone over $\mathrm{Au} / \mathrm{ZrO} 2$ Catalyst Combined with ZSM-5. ACS Catal. 6, 2035-2042. doi:10.1021/ acscatal.5b02882

Conflict of Interest: The authors declare that the research was conducted in the absence of any commercial or financial relationships that could be construed as a potential conflict of interest.

Publisher's Note: All claims expressed in this article are solely those of the authors and do not necessarily represent those of their affiliated organizations, or those of the publisher, the editors, and the reviewers. Any product that may be evaluated in this article, or claim that may be made by its manufacturer, is not guaranteed or endorsed by the publisher.

Copyright $\odot 2021 \mathrm{Hu}, \mathrm{Xu}, \mathrm{Wu}$, Zhang, Zhao, Wang and Yan. This is an open-access article distributed under the terms of the Creative Commons Attribution License (CC $B Y)$. The use, distribution or reproduction in other forums is permitted, provided the original author(s) and the copyright owner(s) are credited and that the original publication in this journal is cited, in accordance with accepted academic practice. No use, distribution or reproduction is permitted which does not comply with these terms. 